\title{
Nucleon-deuteron scattering with $\Delta$-isobar excitation: Perturbation theory
}

\author{
A. Deltuva, ${ }^{*}$ K. Chmielewski, and P. U. Sauer \\ Institut für Theoretische Physik, Universität Hannover, D-30167 Hannover, Germany
}

(Received 5 December 2002; published 23 May 2003)

\begin{abstract}
A perturbative approach for the description of elastic and inelastic nucleon-deuteron scattering is developed. Its validity is discussed. The aim of the perturbative approach is the isolation of details of different reaction mechanisms. The dynamics is based on a two-baryon potential allowing for the excitation of a nucleon to a $\Delta$ isobar. The coupled-channel potential yields an effective three-nucleon force in three-nucleon scattering. The purely nucleonic reference potential is the charge-dependent CD-Bonn potential.
\end{abstract}

DOI: 10.1103/PhysRevC.67.054004 PACS number(s): 21.45.+v, 21.30. $-\mathrm{x}, 24.70 .+\mathrm{s}, 25.10 .+\mathrm{s}$

\section{INTRODUCTION}

We embarked on the description of nucleon-deuteron scattering with $\Delta$-isobar excitation. Reference [1] developed a coupled-channel formulation within the framework of nonrelativistic quantum mechanics: Three-nucleon channels are coupled to those in which one nucleon is turned into a single $\Delta$ isobar. The $\Delta$ isobar is considered a stable baryon with spin and isospin $\frac{3}{2}$. The description applies to scattering energies well below the pion-production threshold. The virtual excitation of the $\Delta$ isobar yields an effective three-nucleon force, besides other $\Delta$-isobar effects.

First results of the coupled-channel description of nucleon-deuteron scattering are given in Refs. [2,3] for elastic scattering, in Ref. [4] for breakup, and in Refs. [5,6] for electromagnetic reactions of the three-nucleon system. Whereas a separable expansion of the two-baryon transition matrix was used in those early calculations, Ref. [7] solves the three-particle scattering equations exactly by Chebyshev expansion of the two-baryon transition matrix as interpolation technique. This technique is found to be highly efficient and systematic. We are proud of all the calculational achievements: The developed theoretical apparatus is based on realistic interactions, the computations are technically sound, and the account of the existing experimental data is surprisingly good. However, in one respect the given description of nucleon-deuteron scattering is highly disappointing: The description does not allow for an immediate physics understanding of the predictions, since the steps from the baryonic interaction, the calculational input, to the details of observables with and without polarization is clouded by highly complicated numerics.

We admit that we are unable to change that situation of theoretical intransparency; this situation is not particular for our calculational scheme; it appears to be the fate of the physics of few-nucleon systems. However, this paper is meant to supply a tool that may help to facilitate the physics understanding of three-nucleon scattering, at least in part and at least for particular situations. The tool is perturbation theory of high calculational accuracy.

\footnotetext{
*On leave from Institute of Theoretical Physics and Astronomy, Vilnius University, Vilnius 2600, Lithuania. Email address: deltuva@itp.uni-hannover.de
}

Section II describes our perturbative approach to the scattering theory of the three-nucleon system; the section formulates the technical apparatus. Section III discusses its range of applicability and tests its validity; the validity will turn out to be quite satisfactory. We use the perturbative approach for $\Delta$-isobar coupling, for charge dependence and for higher partial waves of the two-baryon coupled-channel interaction. Section IV gives a summary.

\section{GENERAL FORMALISM OF PERTURBATION THEORY}

We describe nucleon-deuteron scattering in a three-baryon Hilbert space which has a purely nucleonic sector $\mathcal{H}_{N}$ and a sector $\mathcal{H}_{\Delta}$ in which one nucleon is replaced by a $\Delta$ isobar. The notation is taken over from Ref. [1]; it will only be explained, where necessary for understanding. The dynamics is based on the two-baryon coupled-channel potential derived from the charge-dependent CD-Bonn potential [8] as purely nucleonic reference according to the construction technique of Ref. [9]. The charge dependence carries over to the coupled-channel potential with $\Delta$-isobar excitation; $\Delta$-isobar coupling and charge dependence occur in the same isospin-triplet partial waves. The standard exact calculations, whose results are to be approximated in perturbation theory, include the interaction up to two-baryon total angular momentum $I=5, \Delta$-isobar coupling up to $I=4$, and they employ three-particle partial-wave channels up to three-baryon total angular momentum $\mathcal{J}=\frac{31}{2}$ with three-baryon total isospin $\mathcal{T}=\frac{1}{2}$ and $\frac{3}{2}$. Of course, the number of three-particle partial waves required to obtain converged results depends on the available scattering energy, e.g., at 13, 65, 135, and 190 $\mathrm{MeV}$ nucleon lab energy, partial waves up to three-baryon total angular momentum $\mathcal{J}=\frac{21}{2}, \frac{25}{2}, \frac{27}{2}$, and $\frac{31}{2}$, respectively, are needed. The standard calculations, exact with respect to three-particle dynamics, neglect the rather unimportant coupling between the three-baryon states $\mathcal{T}=\frac{1}{2}$ and $\mathcal{T}=\frac{3}{2}$ arising from the charge dependence of the interaction in higher isospin-triplet waves; they only allow for the coupling due to charge dependence in ${ }^{1} S_{0}$ and ${ }^{3} P_{I}$ waves; the full treatment of $\mathcal{T}=\frac{3}{2}$ in $P$ waves is an improvement compared with Ref. [7]; it yields only minimal and physically irrelevant changes in the breakup spin observables shown there.

The description of nucleon-deuteron scattering is based on the AGS version [10] of three-particle scattering theory. 
The symmetrized multichannel transition matrix between two-body channels $U(Z)$ and the symmetrized breakup transition matrix $U_{0}(Z)$ are given in Ref. [1], i.e.,

$$
\begin{gathered}
U=P G_{0}^{-1}+P T G_{0} U, \\
U_{0}=(1+P) G_{0}^{-1}+(1+P) T G_{0} U .
\end{gathered}
$$

In Eqs. (1), $T$ is the two-baryon transition matrix in threebaryon space, $G_{0}$ the free resolvent $\left(Z-H_{0}\right)^{-1}, H_{0}$ being the free Hamiltonian including rest masses, and $P=P_{123}$ $+P_{132}$ the sum of the cyclic and anticyclic permutation operators of three particles. The term $(1+P) G_{0}^{-1}$ does not contribute to the on-shell matrix elements of $U_{0}$ needed for breakup observables. We leave out the dependence of operators on the three-particle available energy $Z$ and the pair subscript of the two-baryon transition matrix in the notation of this paper, since they are not essential for our considerations, and the equations become more compact by that omission. The three-particle scattering equations (1) are solved exactly with the interpolation technique based on the Chebyshev expansion of the two-baryon transition matrix $T$ according to Ref. [7].

In the following, we develop a perturbative approach which takes into account one part of the interaction exactly and the other part approximately. We decompose the twobaryon and three-baryon transition matrices into two parts, i.e., in Eqs. (1) we replace $T$ by $T+\Delta T, U$ by $U+\Delta U$, and $U_{0}$ by $U_{0}+\Delta U_{0}$. Here, $U$ and $U_{0}$ are defined to be exact solutions of the three-particle scattering equations (1) with the unperturbed part $T$ of the two-baryon transition matrix. The remaining corrections $\Delta U$ and $\Delta U_{0}$ obey the following exact equations:

$$
\begin{gathered}
\Delta U=P T G_{0} \Delta U+P \Delta T G_{0}(U+\Delta U), \\
\Delta U_{0}=(1+P)\left[T G_{0} \Delta U+\Delta T G_{0}(U+\Delta U)\right] .
\end{gathered}
$$

The decompositions (2) can provide meaningful approximations when the contribution of $\Delta T$ to the full multichannel transition matrices is small compared to that of $T$. In this case one can expect that $\Delta U$ and $\Delta U_{0}$ will be small and already their lowest-order approximations will account quite well for the required corrections to $U$ and $U_{0}$, respectively. We therefore solve Eqs. (2) approximately by iteration, i.e.,

$$
\begin{gathered}
\Delta U^{(n)}=P T G_{0} \Delta U^{(n)}+P \Delta T G_{0}\left(U+\Delta U^{(n-1)}\right) \\
=U G_{0} \Delta T G_{0}\left(U+\Delta U^{(n-1)}\right), \\
\Delta U_{0}^{(n)}=(1+P)\left[T G_{0} \Delta U^{(n)}+\Delta T G_{0}\left(U+\Delta U^{(n-1)}\right)\right],
\end{gathered}
$$

with $\Delta U^{(0)}=\Delta U_{0}^{(0)}=0$. The exact solutions of Eqs. (2) are recovered in the limit $\Delta U=\lim _{n \rightarrow \infty} \Delta U^{(n)}$ and $\Delta U_{0}$ $=\lim _{n \rightarrow \infty} \Delta U_{0}^{(n)}$. We emphasize that we do not give the terms small and large (used in connection with the operators $\Delta T, \Delta U$, and $\left.\Delta U_{0}\right)$ a rigorous mathematical meaning.

Equation (3a) is an integral equation for $\Delta U^{(n)}$, analogous to that for $U$ : Both equations have the same kernel, containing the unperturbed part $T$ of the two-baryon transition matrix, only the driving terms being different. That integral equation should be solved, when the half-shell elements of $\Delta U^{(n)}$ are used to compute the corresponding breakup transition matrix $\Delta U_{0}^{(n)}$ according to Eq. (3c). If one is interested in the on-shell elements of $\Delta U^{(n)}$ only, needed for elastic nucleon-deuteron scattering, then quadrature (3b) is more convenient. The numerical solutions of Eqs. (3) use the Chebyshev expansion of the perturbation $\Delta T$ in the same way as the solution of the full scattering equation uses the Chebyshev expansion of the unperturbed transition matrix $T$. We admit that Eqs. (3) are not simpler to solve than those for the corresponding exact dynamics; nevertheless, we hope the perturbative approach to enable us to isolate important physical mechanisms better.

The perturbative approach developed in this section will be applied in the following to particular physics cases. We will always use the lowest approximation order $n$ in which the studied physics effect shows up.

\section{VALIDITY OF PERTURBATION THEORY}

In order to check the reliability of the developed perturbative approach, we compare results for nucleon-deuteron scattering observables obtained with the technique of this paper to the corresponding results of an exact calculation. We do so for both elastic and inelastic scattering. The definitions of observables are given in Refs. [2,4]. The step from the calculated perturbed transition matrices $U+\Delta U$ and $U_{0}$ $+\Delta U_{0}$ to the respective observables is done without any further approximation as in the full calculations. Of course, the predominant perturbative correction of observables is linear in $\Delta U$ and $\Delta U_{0}$; those linear relations are most useful for a qualitative understanding, as demonstrated in Figs. 4 and 6. In general, however, we choose to keep also the quadratic contributions of $\Delta U$ and $\Delta U_{0}$ to the observables; this choice turns out to improve the reliability of the perturbative approach. Though this choice appears to be somehow inconsistent with respect to the orders of perturbation, it seems to minimize a violation of the optical theorem, a fact not fully understood by us. The optical theorem connects linear and quadratic terms of scattering amplitudes in a delicate form, and a violation of it is inherent in most perturbative approaches.

\section{A. $\Delta$-isobar degrees of freedom}

The effect of the $\Delta$ isobar on observables of elastic and inelastic nucleon-deuteron scattering is studied in Refs. $[2-4,7]$ exactly and is found to be quite small. We therefore expect that it can be accounted for well using the perturbative approach as discussed in Sec. II.

The employed two-baryon coupled-channel potential displayed in Fig. 1 yields a corresponding transition matrix whose components $T_{\Delta N}$ and $T_{N \Delta}$ couple the two Hilbert sectors in an obvious notation or act in the additional Hilbert sector $\mathcal{H}_{\Delta}$ through $T_{\Delta \Delta}$. But it also modifies the two-nucleon transition matrix within the purely nucleonic Hilbert sector by $\Delta T_{N N}$ compared with $T_{N N}$, the two-nucleon transition 


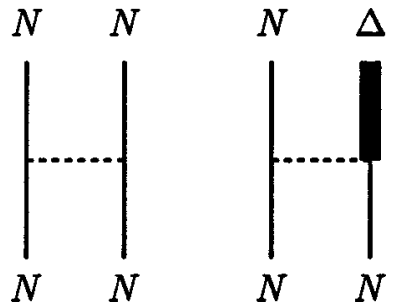

FIG. 1. Two-baryon coupled-channel potential. The Hermitian conjugate of the transition process is not shown. The perturbation theory remains unchanged, if the coupled-channel potential also contains an additional direct part in the Hilbert sector $\mathcal{H}_{\Delta}$.

matrix derived from the purely nucleonic reference potential. In the notation of Sec. II, we write the full coupled-channel two-baryon transition matrix $T+\Delta T$ as

$$
T+\Delta T=T_{N N}+\Delta T_{N N}+T_{\Delta N}+T_{N \Delta}+T_{\Delta \Delta} .
$$

We identify $T_{N N}$ with the unperturbed transition matrix $T$, the corresponding multichannel three-nucleon transition matrices being

$$
\begin{gathered}
U_{N N}=P G_{0}^{-1}+P T_{N N} G_{0} U_{N N}, \\
U_{0 N N}=(1+P) G_{0}^{-1}+(1+P) T_{N N} G_{0} U_{N N} .
\end{gathered}
$$

The additional components of the two-baryon transition matrix $\Delta T_{N N}, T_{\Delta N}, T_{N \Delta}$, and $T_{\Delta \Delta}$ are generated by $\Delta$-isobar excitation and are therefore contained in $\Delta T$. The computation of $\Delta T$ is described in the Appendix. We note that the components $T_{\Delta N}, T_{N \Delta}$, and $T_{\Delta \Delta}$ are in general not small quantities in comparison with $T_{N N}$; this fact is borne out in Fig. 5 of Ref. [1]. However, they enter the expressions for physical amplitudes together with the free resolvent $G_{0}$ in $\Delta$-isobar channels, which suppresses the contribution of the $\Delta$ isobar at the considered scattering energies quite strongly, i.e., the dimensionless operators $G_{0} T_{\Delta N}$ and $T_{N \Delta} G_{0}$ can be treated as small quantities in comparison with $T_{N N} G_{0}$; on those small quantities the perturbative approach is based. $T_{\Delta \Delta}$ shows up in the combination $G_{0} T_{\Delta \Delta} G_{0}$ and therefore contributes in higher order only. In contrast to $T_{N \Delta}, T_{\Delta N}$, and $T_{\Delta \Delta}$, the additional component $\Delta T_{N N}$ of the coupledchannel transition matrix is small in comparison with $T_{N N}$ according to the Appendix.

In order to calculate the observables of elastic and inelastic nucleon-deuteron scattering in the lowest physically relevant order, only the components $\Delta U_{N N}^{(2)}, \Delta U_{0 N N}^{(2)}$ and as an intermediate quantity $\Delta U_{\Delta N}^{(1)}$ are needed, i.e.,

$$
\begin{gathered}
\Delta U_{\Delta N}^{(1)}=P T_{\Delta N} G_{0} U_{N N} \\
\Delta U_{N N}^{(2)}=P T_{N N} G_{0} \Delta U_{N N}^{(2)}+P \Delta T_{N N} G_{0} U_{N N}+P T_{N \Delta} G_{0} \Delta U_{\Delta N}^{(1)} \\
=U_{N N} G_{0}\left(\Delta T_{N N}+T_{N \Delta} G_{0} P T_{\Delta N}\right) G_{0} U_{N N} \\
\Delta U_{0 N N}^{(2)}=(1+P)\left(T_{N N} G_{0} \Delta U_{N N}^{(2)}+\Delta T_{N N} G_{0} U_{N N}\right. \\
\left.+T_{N \Delta} G_{0} \Delta U_{\Delta N}^{(1)}\right)
\end{gathered}
$$

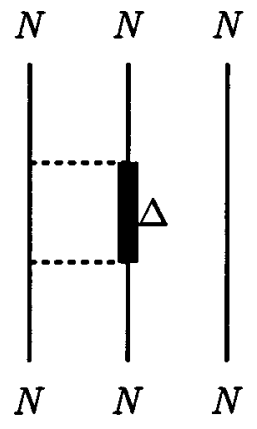

(a)

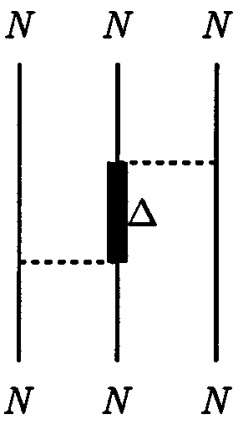

(b)
FIG. 2. Three-nucleon processes taken into account in perturbation theory. Process (a) yields the two-nucleon dispersive effect described by $\Delta T_{N N}$ in Eqs. (6c) and (6e); process (b) yields the effective three-nucleon force by $T_{N \Delta} G_{0} P T_{\Delta N}$.

$$
\begin{aligned}
= & (1+P)\left[T_{N N} G_{0} \Delta U_{N N}^{(2)}\right. \\
& \left.+\left(\Delta T_{N N}+T_{N \Delta} G_{0} P T_{\Delta N}\right) G_{0} U_{N N}\right] .
\end{aligned}
$$

When deriving Eqs. (6) iteratively according to Eqs. (3), the term $P \Delta T_{N N} G_{0} \Delta U_{N N}^{(1)}=P \Delta T_{N N} G_{0} U_{N N} G_{0} \Delta T_{N N} G_{0} U_{N N}$ is neglected as being of second order in the small quantity $\Delta T_{N N}$; all remaining terms in Eqs. (6b)-(6e) are of first order in the corresponding small quantities; the used label $n=2$ is technical in the iterative spirit of Eqs. (3), it does not reflect the order of small quantities.

In Eqs. (6), one can clearly see two different $\Delta$-isobar effects, i.e., the two-nucleon dispersion and the effective three-nucleon force, described by the first and second terms proportional to $\Delta T_{N N}$ and to $T_{N \Delta} G_{0} P T_{\Delta N}$ in Eqs. (6c) and (6e), respectively. The corresponding characteristic processes are shown in Fig. 2.

As found in Refs. [2-4,7], the $\Delta$-isobar effect is more pronounced at higher scattering energies. In Figs. 3 and 4 we therefore present a comparison for selected observables at higher energies only, i.e., for nucleon-deuteron breakup scattering at $65 \mathrm{MeV}$ nucleon lab energy and for elastic nucleondeuteron scattering at $135 \mathrm{MeV}$ nucleon lab energy. Besides the full calculation, a perturbative one is carried out, the unperturbed interaction being the purely nucleonic reference potential and $\Delta$-isobar coupling being considered the perturbation. Even on the fine scale of the rather moderate $\Delta$-isobar effect to the considered observables, the perturbative treatment of $\Delta$-isobar coupling is reliable. The observables shown in Figs. 3 and 4 are characteristic for all calculated ones. In addition, Fig. 4 separates the two different $\Delta$-isobar effects proportional to $\Delta T_{N N}$ and $T_{N \Delta} G_{0} P T_{\Delta N}$ in Eq. (6c); the general competition between those two effects, typical for trinucleon binding and low-energy scattering observables $[2,7]$, does not remain so pronounced for observables at higher energies.

\section{B. Charge dependence}

The effect of charge dependence in the two-baryon interaction on observables of nucleon-deuteron scattering was calculated exactly by us [7] and in Ref. [13], and was found 

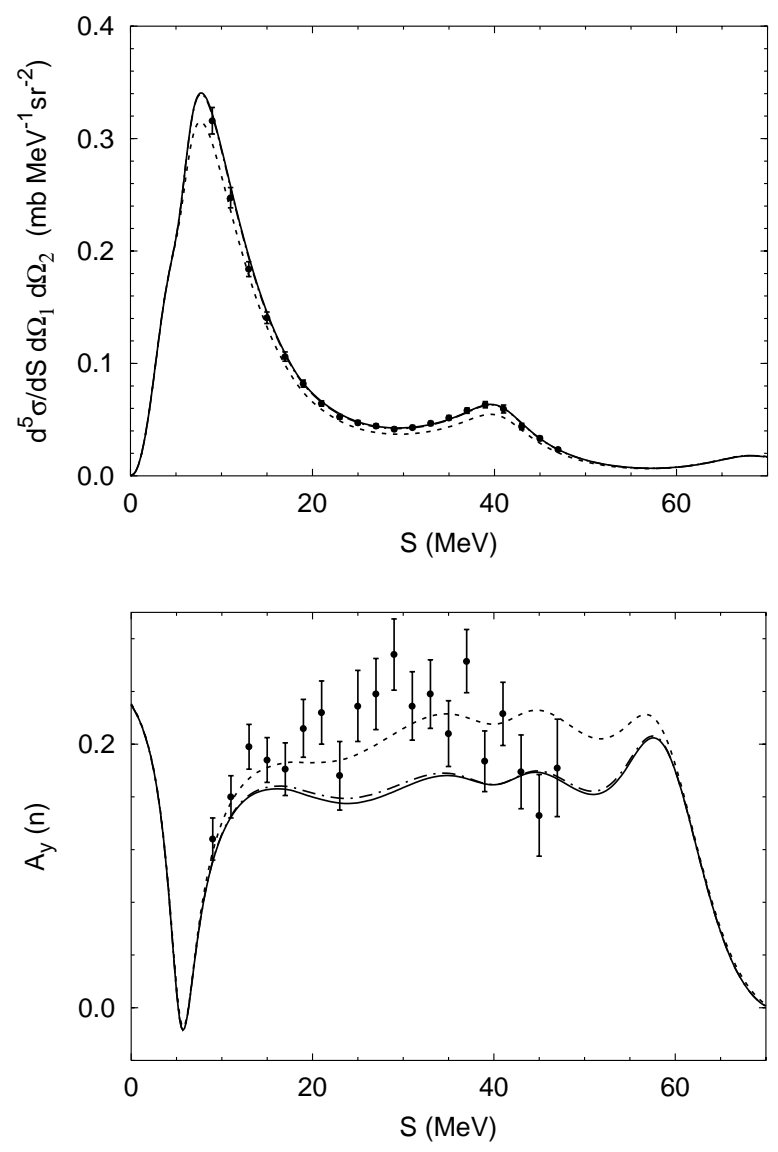

FIG. 3. Differential cross section and nucleon analyzing power $A_{y}(n)$ of nucleon-deuteron breakup as function of the arclength $S$ along the kinematical curve at $65 \mathrm{MeV}$ nucleon lab energy in the collinearity configuration $\left(30^{\circ}, 98^{\circ}, 180^{\circ}\right)$. Results of the perturbative treatment of the $\Delta$ isobar (dash-dotted curves) are compared with results of the exact calculation with the $\Delta$ isobar (solid curves); they cannot be differentiated in the upper observable and only slightly in the lower one. In order to appreciate the size of the $\Delta$-isobar effect to be perturbatively accounted for, results of a calculation with the purely nucleonic reference potential are also given as dashed curves. The experimental data are from Ref. [11] and refer to proton-deuteron scattering.

to be quite small, except in special kinematic situations of breakup. We therefore expect that it can be accounted for well using the perturbative approach as discussed in Sec. II.

Older force models for the two-nucleon interaction assume charge independence; i.e., in the isospin-triplet partial waves, proton-proton $(p p)$, neutron-proton $(n p)$, and neutron-neutron $(n n)$ potentials are taken to be the same. The three-nucleon bound state and the nucleon-deuteron scattering states, described without Coulomb interaction, only have wave function components with total isospin $\mathcal{T}$ $=\frac{1}{2}$. Charge dependence, allowed for in modern potentials such as CD-Bonn potential, changes the wave function components with total isospin $\mathcal{T}=\frac{1}{2}$ and develops additional components with total isospin $\mathcal{T}=\frac{3}{2}$. This section tries to study those changes due to charge dependence perturbatively.

Neutron-deuteron scattering is considered. The twobaryon transition matrix $T$ in three-particle Hilbert space has components $T_{2 \mathcal{T}^{\prime} 2 \mathcal{T}}$ with respect to total isospin $\mathcal{T}[7,13]$. The charge-independent starting point does not couple total isospin, i.e., $T=T_{11}+T_{33}$; we assume the proton-proton interaction to be representative for the complete isospin triplets, and therefore $T_{11}=T_{33}=T_{p p}$. We take the protonproton interaction as charge-independent reference, since most charge-independent potentials such as the Paris potential [14] were based on proton-proton data, the exception being the family of original Bonn potentials [15] which were tuned to neutron-proton data. Of course, the actual intermediate effects of charge dependence are different when the neutron-proton interaction is used as charge-independent reference.

We take the CD-Bonn potential, extended to $\Delta$-isobar excitation, as example for a charge-dependent potential; its full charge-dependent transition matrix has the form

$$
T+\Delta T=T_{11}+T_{33}+\Delta T_{11}+\Delta T_{31}+\Delta T_{13}+\Delta T_{33} .
$$

We identify $T_{11}+T_{33}$ with the unperturbed transition matrix $T$, the corresponding multichannel transition matrices being

$$
\begin{gathered}
U_{11}=P G_{0}^{-1}+P T_{11} G_{0} U_{1}, \\
U_{011}=(1+P) G_{0}^{-1}+(1+P) T_{11} G_{0} U_{11} .
\end{gathered}
$$

The notation $U_{2 \mathcal{T}^{\prime} 2 \mathcal{T}}$ and $U_{02} \mathcal{T}^{\prime} 2 \mathcal{T}$ makes the possible couplings of total isospin explicit. The initial nucleon-deuteron channel state has total isospin $\mathcal{T}=\frac{1}{2}$; the charge-independent interaction cannot couple to another total isospin component.

Charge dependence introduces isospin coupling into the two-baryon transition matrix as indicated by $\Delta T_{2 \mathcal{T}^{\prime} 2 \mathcal{T}}$ in Eq. (7). The arising perturbing components have the following explicit forms:

$$
\begin{gathered}
\Delta T_{11}=\frac{2}{3} T_{n n}+\frac{1}{3} T_{n p}-T_{p p}, \\
\Delta T_{31}=\frac{\sqrt{2}}{3}\left(T_{n p}-T_{n n}\right), \\
\Delta T_{13}=\Delta T_{31}, \\
\Delta T_{33}=\frac{1}{3} T_{n n}+\frac{2}{3} T_{n p}-T_{p p}
\end{gathered}
$$

for neutron-deuteron scattering. The perturbing components $\Delta T_{2 \mathcal{T}^{\prime} 2 \mathcal{T}}$ can be considered to be small quantities compared with the unperturbed $T$. In order to calculate the observables of elastic and inelastic neutron-deuteron scattering in the lowest physically relevant order, only the components $\Delta U_{11}^{(1)}$ for elastic scattering, $\Delta U_{02 \mathcal{T}^{\prime} 1}^{(1)}$ for breakup, and $\Delta U_{31}^{(1)}$ as an intermediate quantity are needed; i.e.,

$$
\begin{aligned}
\Delta U_{11}^{(1)} & =P T_{11} G_{0} \Delta U_{11}^{(1)}+P \Delta T_{11} G_{0} U_{11} \\
& =U_{11} G_{0} \Delta T_{11} G_{0} U_{11}, \\
\Delta U_{31}^{(1)} & =P T_{33} G_{0} \Delta U_{31}^{(1)}+P \Delta T_{31} G_{0} U_{11},
\end{aligned}
$$



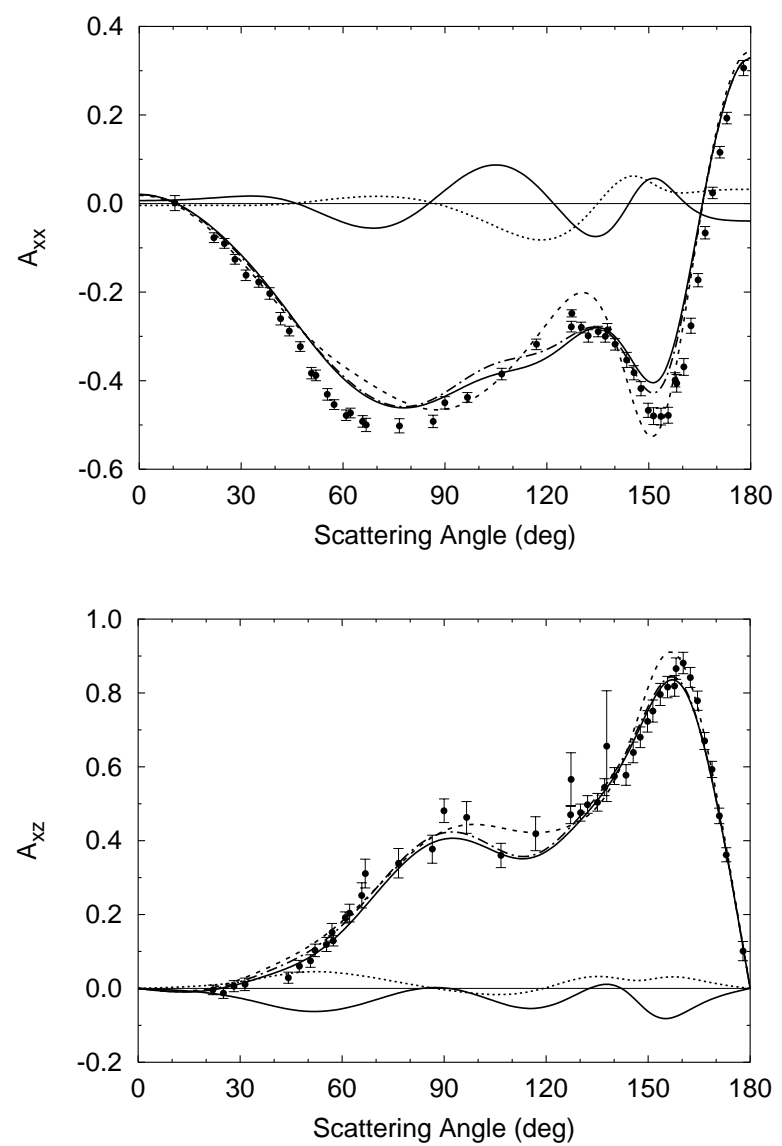

FIG. 4. The two- and three-nucleon force effects of the $\Delta$ isobar for the deuteron analyzing powers $A_{x x}$ and $A_{x z}$ in elastic nucleondeuteron scattering as function of the center of mass (c.m.) scattering angle at $135 \mathrm{MeV}$ nucleon lab energy. The separated effects are shown by the two curves around the horizontal zero line. There, the dotted (solid) curves refer to the effective two-nucleon (threenucleon) contributions to the observables according to Eq. (6c); they are calculated up to first order in $\Delta U$ for illustrative reasons as discussed in the first paragraph of Sec. III, and they therefore do not sum up precisely to the full perturbative result, as used by us. The full results of $A_{x x}$ and $A_{x z}$ are also given. The perturbative results (dash-dotted curves) almost coincide with the results of exact calculations (solid curves). Results without $\Delta$-isobar excitation (dashed curves) are given as reference for the complete $\Delta$-isobar effect. The experimental data are from Ref. [12] and refer to protondeuteron scattering.

$$
\Delta U_{02 \mathcal{T}^{\prime} 1}^{(1)}=(1+P)\left(T_{2 \mathcal{T}^{\prime} 2 \mathcal{T}^{\prime}} G_{0} \Delta U_{2 \mathcal{T}^{\prime} 1}+\Delta T_{2 \mathcal{T}^{\prime} 1} G_{0} U_{11}\right) .
$$

It is best to use Eq. (10b) for the on-shell correction $\Delta U_{11}^{(1)}$ of elastic neutron-deuteron scattering. The on-shell breakup correction has transitions to total isospin $\mathcal{T}^{\prime}=\frac{3}{2}$; quadrature (10d) for that correction requires half-shell elements of the two components $\Delta U_{2 \mathcal{T}^{\prime} 1}$; they are best calculated from the integral equations (10a) and (10c). The effect of charge dependence is most pronounced at low energies; there the simultaneous $\Delta$-isobar effect is negligible and will not be discussed in this section anymore.
Observables of elastic scattering are not documented in the figures. For them the effect of charge dependence is very small, this is also for the sensitive nucleon analyzing power $A_{y}(n)$ at $10 \mathrm{MeV}$ nucleon lab energy $[7,13]$. It is perfectly accounted for by perturbation theory and decreases with increasing energy. Furthermore, according to Eq. (10b) only total isospin $\mathcal{T}=\frac{1}{2}$ states contribute to corrections of elastic scattering in lowest order of perturbation theory.

Observables of breakup at $13 \mathrm{MeV}$ nucleon lab energy are shown in Fig. 5. Even on the fine scale of the moderate effect of charge dependence, the perturbative treatment of charge dependence is highly reliable. The full treatment of charge dependence is crucial for the differential cross sections in the vicinity of final-state interaction (FSI) peaks, as already pointed out in Ref. [13]. In our description, as illustrated in detail in Fig. 6, left side, about $\frac{2}{3}$ of the effect of charge dependence in the neutron-proton FSI peak is due to the coupling to total isospin $\mathcal{T}=\frac{3}{2}$, only $\frac{1}{3}$ due to changes in $\Delta T_{11}$; the effect arises almost exclusively from the charge dependence in the ${ }^{1} S_{0}$ two-baryon partial wave. The effect of charge dependence in the isospin-triplet $P$ waves is negligible for the differential cross section; however, it contributes to the smaller effects in characteristic spin observables.

The ratio $\frac{2}{3}: \frac{1}{3}$ can be explained in the following way: The $n p$ FSI should be described rather well-and, in fact, it isusing the $n p$ potential for all two-nucleon interactions without any charge dependence. Thus, when starting out with a $p p$ potential for all, the correction $T_{n p}-T_{p p}$ has to be applied. However, allowing for charge dependence but keeping charge symmetry, Eq. (9a) yields $\Delta T_{11}=\frac{1}{3}\left(T_{n p}-T_{p p}\right)$; according to Eqs. (10a) and (10d) the correction $\Delta U_{011}$ is proportional to $\Delta T_{11}$. Thus, assuming predominant linearity in $\Delta U_{011}$ for the corrections to observables, the changes in the $\mathcal{T}=\frac{1}{2}$ component yield only $\frac{1}{3}$ of the full charge-dependent effect; the $\mathcal{T}=\frac{3}{2}$ component $\Delta U_{031}$ has to yield the remaining part, i.e., $\frac{2}{3}$. Of course, the above consideration is valid only in the vicinity of the $n p$ FSI peak.

Depending on the assumed charge-independent reference, a model-dependent choice, the individual charge-dependent effects are of course different. If, in contrast to the strategy of this section, the neutron-proton interaction is taken as charge-independent representative for all isospin-triplet components, the complete effect of charge dependence on the differential breakup cross section in the neutron-proton FSI kinematics of Fig. 5 is almost zero; the neutron-proton FSI is well described by a charge-independent reference based on a neutron-proton potential [13]. The effect of $\Delta T_{11}$ on the standard total-isospin state $\mathcal{T}=\frac{1}{2}$ and the effect due to the nonstandard $\mathcal{T}=\frac{3}{2}$ component almost cancel each other, though being sizable individually; this fact is borne out in Fig. 6, right side. In fact, the theoretical explanation is the same as the reasoning in the previous paragraph for the previous charge-independent choice, however, $\Delta T_{11}=-\frac{2}{3}\left(T_{n p}-T_{p p}\right)$ in contrast to Eq. (9a); this is why the effect due to $\Delta U_{011}$ is of doubled magnitude and opposite in sign compared to that of the previous paragraph as shown in Fig. 6, left side. Correspondly, in the neutron-neutron FSI regions the complete effect of charge dependence is large, when the neutronproton interaction is the assumed reference; it was small be- 

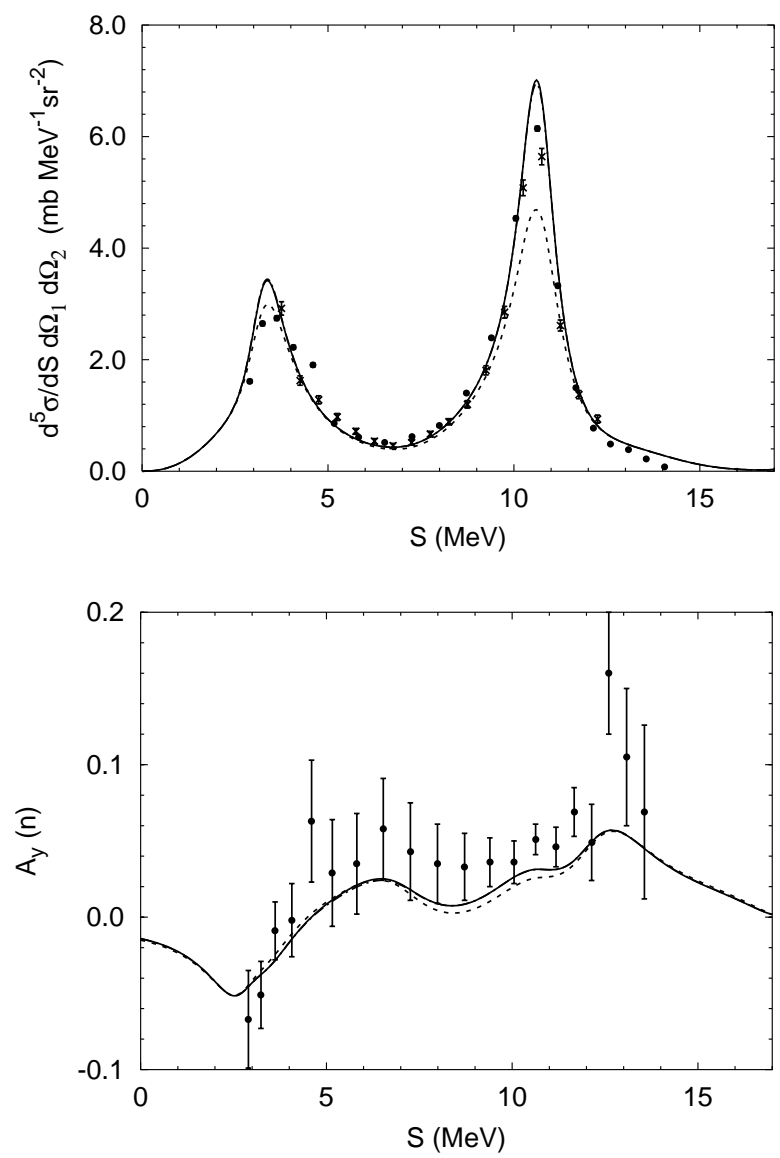

FIG. 5. Differential cross section and nucleon analyzing power $A_{y}(n)$ of nucleon-deuteron breakup as function of the arclength $S$ along the kinematical curve at $13 \mathrm{MeV}$ nucleon lab energy in the neutron-proton FSI configuration $\left(39.0^{\circ}, 62.5^{\circ}, 180.0^{\circ}\right)$. Results of the perturbative treatment of the charge dependence (dash-dotted curves) are compared with results of the exact treatment of the charge dependence (solid curves); they are not distinguishable in both plots. In order to appreciate the size of the effect to be perturbatively accounted for, results of a calculation without charge dependence, i.e., with the proton-proton interaction in all components of the isospin-triplet two-baryon partial waves, are also given as dashed curves. The experimental data are from Ref. [16], referring to neutron-deuteron scattering (crosses), and from Ref. [17], referring to proton-deuteron scattering (full circles).

fore, when the proton-proton interaction was the assumed reference [13]. Of course, irrespective of how the chargeindependent reference is chosen, the full charge-dependent results are always the same; this fact can also be read off from Fig. 6.

\section{Higher two-baryon partial waves}

The contribution to the scattering amplitudes arising from the two-baryon interaction in higher partial waves gets increasingly small. We therefore expect that a perturbative treatment of sufficiently high partial waves is a reliable approximation.

The two-baryon interaction is split into two parts according to the total two-baryon angular momentum $I$ : The inter-
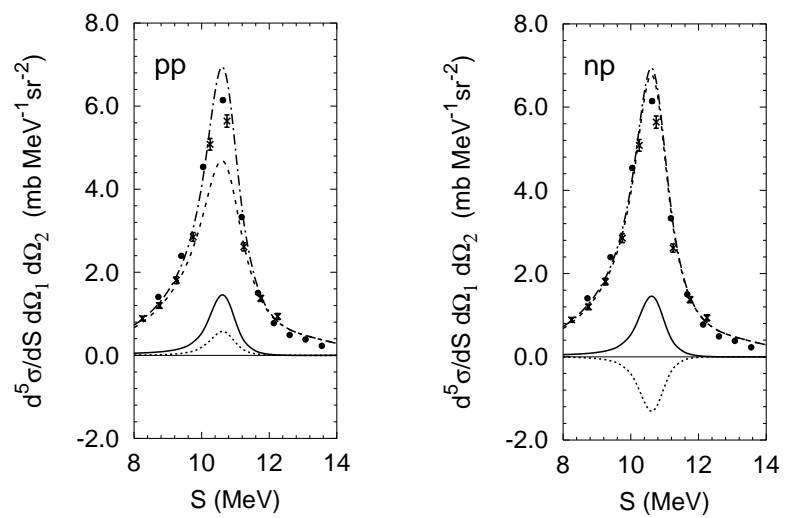

FIG. 6. Model dependence of the charge-dependent effects on the assumed charge-independent reference. The effects on the differential cross section of nucleon-deuteron breakup as function of the arclength $S$ along the kinematical curve at $13 \mathrm{MeV}$ nucleon lab energy in the neutron-proton FSI configuration $\left(39.0^{\circ}, 62.5^{\circ}, 180.0^{\circ}\right)$, which arise from the nonstandard $\mathcal{T}=\frac{3}{2}$ component in the transition matrix (solid curves) and from changes in the standard $\mathcal{T}=\frac{1}{2}$ component (dotted curves), are shown; they are calculated up to first order in $\Delta U_{0}$ for illustrative reasons as discussed in the first paragraph of Sec. III, and they therefore do not sum up precisely to the full perturbative result, as used by us. The left figure corresponds to the proton-proton interaction as chargeindependent reference, the right figure to the neutron-proton interaction as charge-independent reference. Full results of the perturbative treatment of charge dependence (dash-dotted curves) and results of a calculation without charge dependence (dashed curves) are also given; they almost coincide in the right figure. The experimental data are from Ref. [16], referring to neutron-deuteron scattering (crosses), and from Ref. [17], referring to proton-deuteron scattering (full circles).

action in the lower two-baryon partial waves consists of those with $I \leqslant I_{l}$, and the interaction in the higher twobaryon partial waves of those with $I_{l}<I \leqslant I_{h}, I_{h}$ being large enough to accommodate all significant dynamic aspects of the considered reaction. The additional condition $I_{l} \geqslant 1$ guarantees that the interaction in the asymptotic nucleondeuteron states belongs to the lower partial waves. The components of the two-baryon transition matrix in lower and higher partial waves are $T_{l}$ and $T_{h}$, respectively. In our perturbative approach of Eqs. (3), we choose the unperturbed transition matrix $T$ to be $T_{l}$ and the perturbing part $\Delta T$ to be $T_{h}$, i.e.,

$$
T+\Delta T=T_{l}+T_{h} .
$$

The solutions of the scattering equations (1) for the threebaryon transition matrices with the two-baryon interaction $T_{l}$ in lower two-baryon partial waves only are

$$
\begin{gathered}
U_{l}=P G_{0}^{-1}+P T_{l} G_{0} U_{l}, \\
U_{0 l}=(1+P) G_{0}^{-1}+(1+P) T_{l} G_{0} U_{l} .
\end{gathered}
$$

The permutation operator $P$ couples three-baryon states with lower and higher two-baryon quantum numbers $I$. Thus, the three-baryon transition matrices $U_{l}$ and $U_{0 l}$ also have non- 
vanishing components with lower and higher two-baryon angular momenta $I$. The components of $U_{l}$ in partial waves with lower $I$ are obtained from integral equation (12a), the components describing the transitions to partial waves with higher $I$ are derived from the same equation (12a) by quadrature. We need the latter components for the perturbative approach.

The perturbing part of the two-baryon transition matrix is $T_{h} ; T_{h}$ is considered small compared to $T_{l}$. Thus, the approximations for $\Delta U$ and $\Delta U_{0}$ up to first order in $T_{h}$ are

$$
\begin{gathered}
\Delta U^{(1)}=P T_{l} G_{0} \Delta U^{(1)}+P T_{h} G_{0} U_{l} \\
=U_{l} G_{0} T_{h} G_{0} U_{l}, \\
\Delta U_{0}^{(1)}=(1+P)\left(T_{l} G_{0} \Delta U^{(1)}+T_{h} G_{0} U_{l}\right) .
\end{gathered}
$$

With respect to observables of elastic and inelastic nucleondeuteron scattering, it is obviously sufficient to calculate the components of $\Delta U^{(1)}$ only between three-particle partial waves with low two-baryon angular momentum $I$.

We emphasize that in this section the transition matrices $T_{l}$ and $T_{h}$ may refer to a purely nucleonic interaction or to a coupled-channel interaction with coupling of nucleonic states to states with a $\Delta$ isobar. Compared with Sec. III A, the perturbative treatment of the $\Delta$ isobar in the latter case is slightly different. In Sec. III A, terms of the type $T_{N \Delta} G_{0} P T_{\Delta N}$ may contain transition matrices of the higher included partial waves twice, in this section the perturbatively treated transition matrices $T_{\Delta N}$ or $T_{N \Delta}$ of higher partial waves are included in first order only.

The contributions $\Delta U$ and $\Delta U_{0}$ to three-baryon transition matrices arising from the two-baryon interaction in higher partial waves increase with increasing energy. We therefore present a comparison for observables at higher energies only, i.e., for nucleon-deuteron breakup at $65 \mathrm{MeV}$ nucleon lab energy and for elastic nucleon-deuteron scattering at 135 $\mathrm{MeV}$ nucleon lab energy. The comparison is based on a nonvanishing two-baryon interaction in partial waves up to $I$ $=5$, the isospin triplet partial waves up to $I=4$ being coupled to the $\Delta$ isobar. Besides the full calculation, a perturbative one is carried out, the unperturbed interaction being the coupled-channel interaction in two-baryon partial waves up to $I_{l}=2$, the coupled-channel interaction in partial waves with $I_{l}<I \leqslant I_{h}=5$ being considered as the perturbation. Selected results are compared in Fig. 7. The shown agreement between full and perturbative calculations is characteristic for all calculated observables; thus, the perturbative treatment of the interaction in higher two-baryon partial waves is highly reliable.

After the validity of perturbation theory for the higher two-baryon partial waves of the interaction is established, the convergence of theoretical predictions with respect to those higher partial waves for observables of elastic nucleondeuteron scattering and breakup is studied in Fig. 8 for going beyond our standard approach.

Perturbatively, also $I=6$ and $I=7$ partial waves are included, as well as $\Delta$-isobar coupling is extended up to the $I=5$ two-baryon partial waves. On the scale of accuracy
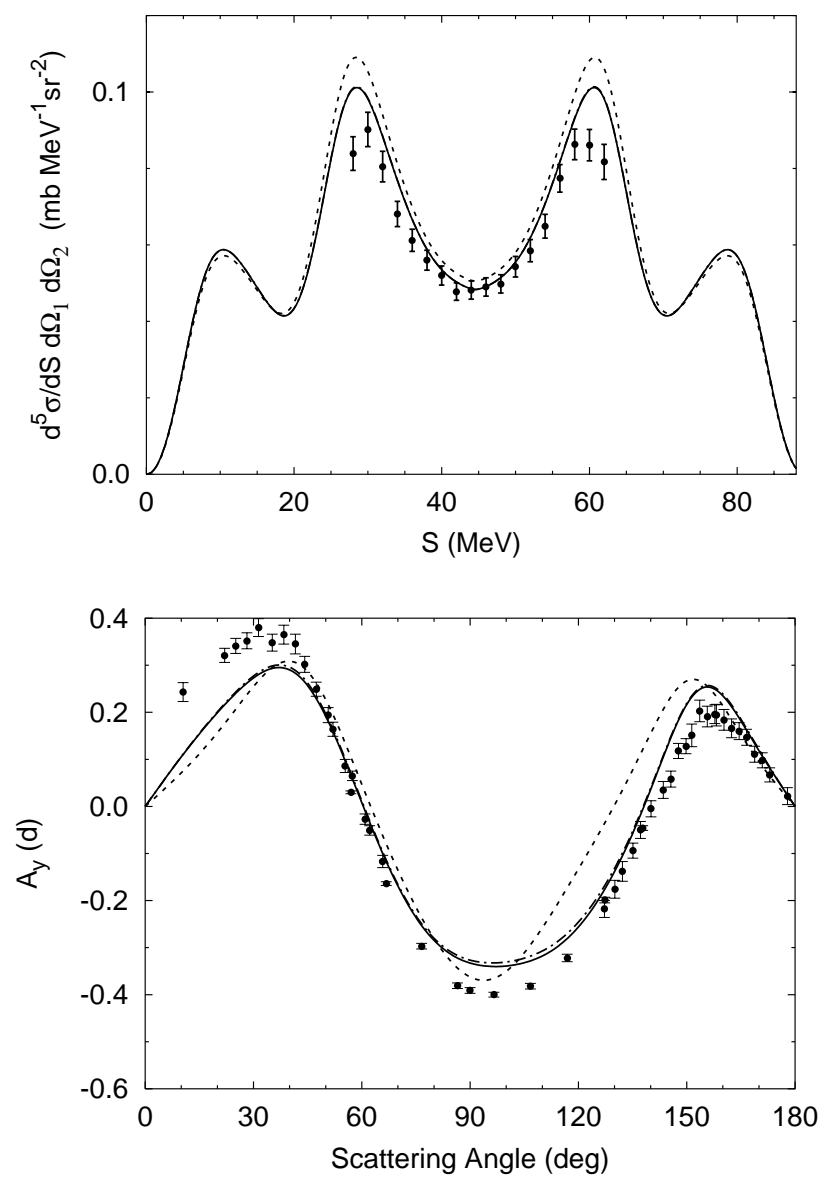

FIG. 7. Differential cross section of nucleon-deuteron breakup as function of the arclength $S$ along the kinematical curve at 65 $\mathrm{MeV}$ nucleon lab energy in the collinearity configuration $\left(59.5^{\circ}, 59.5^{\circ}, 180.0^{\circ}\right)$ and deuteron analyzing power $A_{y}(d)$ of elastic nucleon-deuteron scattering as function of the c.m. scattering angle at $135 \mathrm{MeV}$ nucleon lab energy. Results of the perturbative treatment of the two-baryon partial waves with $3 \leqslant I \leqslant 5$ (dashdotted curves) are compared with results of the exact calculation including two-baryon partial waves up to $I=5$ (solid curves); differences are only discernible in the minimum of $A_{y}(d)$. In order to appreciate the size of the effect to be perturbatively accounted for, results of an exact reference calculation up to $I=2$ are also given as dashed curves. The experimental data are from Refs. [11,12] and refer to proton-deuteron scattering.

required by present-day data, the effect of partial waves $I$ $>5$ and of $\Delta$-isobar coupling for $I>4$ is not discernible in the theoretical predictions. We found only one exception, i.e., the nucleon-to-nucleon spin transfer coefficient $K_{y}^{y}{ }^{\prime}(n n)$ of elastic nucleon-deuteron scattering at $190 \mathrm{MeV}$ nucleon lab energy; it requires partial waves up to $I=6$ for convergence. A sample result for the rapid convergence with respect to higher partial waves is given in Fig. 8; it is characteristic for most studied observables of elastic nucleon-deuteron scattering and breakup; the exceptional case is also given in Fig. 8.

\section{SUMMARY}

Years ago, Ref. [19] developed a perturbative approach for studying properties of the three-nucleon bound state. The 

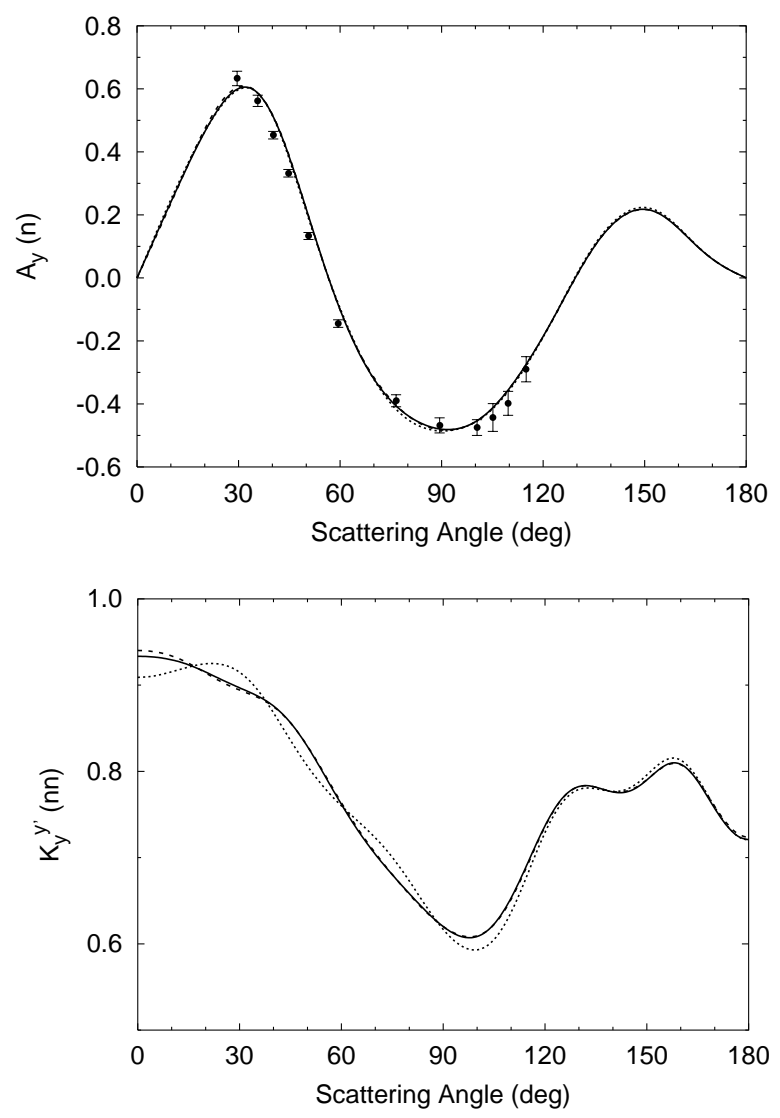

FIG. 8. Nucleon analyzing power $A_{y}(n)$ and nucleon-to-nucleon spin transfer coefficient $K_{y}^{y}{ }^{\prime}(n n)$ of elastic nucleon-deuteron scattering as function of the c.m. scattering angle at $190 \mathrm{MeV}$ nucleon lab energy. Results including the interaction in partial waves up to two-baryon total angular momentum $I=7$ (solid curves), $I=6$ (dashed curves), and $I=5$ (dotted curves) are shown; differences are only discernible for the observable $K_{y}^{y}{ }^{\prime}(n n)$. The experimental data are from Ref. [18] and refer to proton-deuteron scattering.

approach helped to understand effects arising from $\Delta$-isobar excitation better; it also clarified how the perturbative calculation of wave function components with a $\Delta$ isobar and of exchange-current effects arising from the $\Delta$ isobar should be carried out in a reliable fashion. This paper extends and generalizes that approach to nucleon-deuteron scattering, elastically and with breakup.

A perturbative procedure is given for calculating corrections to the multichannel transition matrices $U$ for elastic scattering and $U_{0}$ for breakup due to perturbing contributions in the underlying dynamics. The paper is meant to establish the numerical reliability of that procedure for scattering effects arising from $\Delta$-isobar excitation, from charge dependence and from high partial waves in the two-baryon interaction. The found reliability of the perturbative approach is impressive. It can therefore be used for isolating reaction mechanisms in detail. We summarize our first results as follows.

(1) $\Delta$-isobar excitation in the two-baryon interaction has two distinct effects in the nuclear medium, i.e., it yields an effective two-nucleon repulsion, the two-nucleon dispersion, and an effective three-nucleon force, which is usually attractive. The competition between these two effects on nuclear binding is well known. The competition for observables of elastic and inelastic nucleon-deuteron scattering becomes less pronounced at higher scattering energies.

(2) Charge dependence of the two-nucleon interaction is most important in the ${ }^{1} S_{0}$ partial wave. The difference between the neutron-proton and neutron-neutron ${ }^{1} S_{0}$ interactions is clearly seen in the FSI kinematics of breakup. The charge dependence in the higher isospin-triplet partial waves is less important, but discernible in spin observables of breakup. In addition, there is model dependence in the charge-dependent effects on the assumed charge-independent reference; it is discussed in detail in Sec. III B.

(3) Convergence problems with respect to the inclusion of the interaction in high two-baryon partial waves are studied with the conclusion: Observables of elastic nucleon-deuteron scattering and of nucleon-deuteron breakup about up to the first particle production threshold are sufficiently well described by a two-baryon interaction, nonvanishing in partial waves up to total two-baryon angular momentum $I=5$; coupling to a $\Delta$ isobar has to be included up to partial waves with $I=4$.

We admit freely that the numerics of the perturbative approach is in general not simpler than that of the full calculation. Thus, we do not want to sell the approach of this paper as a substitute for exact calculations to theoreticians with limited computational resources. Furthermore, most reaction mechanisms can also be separated by full calculations with a different dynamic input. However, we still believe that in particular cases the perturbative approach is better able to isolate the smaller effects of individual reaction mechanisms present in the various observables of elastic nucleondeuteron scattering and breakup, and to make their working clearer. In this way we plan to use it in future, as an illuminating complement to exact calculations of three-nucleon scattering.

\section{ACKNOWLEDGMENTS}

The authors are grateful to R. Machleidt for providing them with the computer code for the CD-Bonn potential. A.D. acknowledges a valuable DAAD grant for graduate studies at the University of Hannover. The numerical calculations were performed at the Regionales Rechenzentrum für Niedersachsen.

\section{APPENDIX: TWO-BARYON TRANSITION MATRIX WITH CHANNEL COUPLING}

The two-baryon transition matrix is determined by the Lippmann-Schwinger equation. In the case of a purely nucleonic potential $v_{N N}$, the Lippmann-Schwinger equation takes the form

$$
t_{N N}(z)=v_{N N}+v_{N N} g_{0}(z) t_{N N}(z)
$$

We use lower-case letters when denoting operators in the two-baryon Hilbert space, here the potential $v$, the transition matrix $t(z)$, and the free resolvent $g_{0}(z)$. In contrast to the 
main body of this paper we keep the dependence of operators on the available two-baryon energy $z$ explicitly in this appendix. For the general coupled-channel potential

$$
v+\Delta v=v_{N N}+\Delta v_{N N}+v_{\Delta N}+v_{N \Delta}+v_{\Delta \Delta},
$$

the corresponding components of the two-baryon coupledchannel transition matrix obey the following set of coupled Lippmann-Schwinger equations, i.e.,

$$
\begin{gathered}
t_{N N}(z)+\Delta t_{N N}(z)=\left(v_{N N}+\Delta v_{N N}\right)\left\{1+g_{0}(z)\left[t_{N N}(z)\right.\right. \\
\left.\left.+\Delta t_{N N}(z)\right]\right\}+v_{N \Delta} g_{0}(z) t_{\Delta N}(z), \\
t_{\Delta N}(z)=v_{\Delta N}\left\{1+g_{0}(z)\left[t_{N N}(z)+\Delta t_{N N}(z)\right]\right\} \\
+v_{\Delta \Delta} g_{0}(z) t_{\Delta N}(z), \\
t_{N \Delta}(z)=\left[t_{\Delta N}(z)\right]^{T}, \\
t_{\Delta \Delta}(z)=v_{\Delta \Delta}\left[1+g_{0}(z) t_{\Delta \Delta}(z)\right]+v_{\Delta N} g_{0}(z) t_{N \Delta}(z)
\end{gathered}
$$

Since usually $\Delta v_{N N}$ is small compared to $v_{N N}, \Delta t_{N N}(z)$ is also small compared to $t_{N N}(z)$. The perturbative approach, developed in Eqs. (6) of Sec. III A for $\Delta$-isobar coupling, requires $\Delta t_{N N}(z), t_{\Delta N}(z)$, and $t_{N \Delta}(z)$ translated into threebaryon Hilbert space; $t_{\Delta \Delta}(z)$ is not needed in the order of perturbation theory considered there. Since $\Delta t_{N N}(z)$ is small compared to $t_{N N}(z)$, and $g_{0}(z) t_{\Delta N}(z)$ is small compared to $g_{0}(z) t_{N N}(z)$, the following approximations:

$$
\begin{gathered}
t_{\Delta N}(z)=v_{\Delta N}\left[1+g_{0}(z) t_{N N}(z)\right] \\
t_{N \Delta}(z)=\left[t_{\Delta N}(z)\right]^{T} \\
\Delta t_{N N}(z)=\left[t_{N N}(z) g_{0}(z)+1\right]\left[v_{N \Delta} g_{0}(z) v_{\Delta N}+\Delta v_{N N}\right] \\
\times\left[1+g_{0}(z) t_{N N}(z)\right]
\end{gathered}
$$

are sufficiently accurate for the perturbing components $\Delta T$ of the two-baryon coupled-channel transition matrix, required in Eqs. (6); the approximations (A4) are also consistent with the approximations leading to Eqs. (6).

For the coupled-channel potential of Fig. 1, constructed according to the procedure of Ref. [9], the perturbing potential components are $v_{\Delta N}, \quad v_{N \Delta}=v_{\Delta N}^{\dagger}$, and $\Delta v_{N N}=$ $-v_{N \Delta} g_{0}(0) v_{\Delta N}$. This particular choice of $\Delta v_{N N}$ ensures phase equivalence with the purely nucleonic reference potential $v_{N N}$ at zero energy, i.e., $\Delta t_{N N}(0)=0$.
[1] S. Nemoto, K. Chmielewski, J. Haidenbauer, S. Oryu, P.U. Sauer, and N.W. Schellingerhout, Few-Body Syst. 24, 213 (1998).

[2] S. Nemoto, K. Chmielewski, J. Haidenbauer, U. Meyer, S. Oryu, and P.U. Sauer, Few-Body Syst. 24, 241 (1998).

[3] S. Nemoto, K. Chmielewski, S. Oryu, and P.U. Sauer, Phys. Rev. C 58, 2599 (1998).

[4] K. Chmielewski, A. Deltuva, A.C. Fonseca, S. Nemoto, and P.U. Sauer, Phys. Rev. C 67, 014002 (2003).

[5] L.P. Yuan, K. Chmielewski, M. Oelsner, P.U. Sauer, A.C. Fonseca, and J. Adam, Jr., Few-Body Syst. 32, 83 (2002).

[6] L.P. Yuan, K. Chmielewski, M. Oelsner, P.U. Sauer, and J. Adam, Jr., Phys. Rev. C 66, 054004 (2002).

[7] A. Deltuva, K. Chmielewski, and P.U. Sauer, Phys. Rev. C 67, 034001 (2003).

[8] R. Machleidt, Phys. Rev. C 63, 024001 (2001).

[9] C. Hajduk, P.U. Sauer, and W. Strueve, Nucl. Phys. A405, 581
(1983).

[10] E.O. Alt, P. Grassberger, and W. Sandhas, Nucl. Phys. B2, 167 (1967).

[11] M. Allet et al., Phys. Rev. C 50, 602 (1994).

[12] K. Sekiguchi et al., Phys. Rev. C 65, 034003 (2002).

[13] W. Glöckle, H. Witala, D. Hüber, H. Kamada, and J. Golak, Phys. Rep. 274, 107 (1996).

[14] M. Lacombe, B. Loiseau, J.M. Richard, R. Vinh Mau, J. Coté, P. Pirès, and R. de Tourreil, Phys. Rev. C 21, 861 (1980).

[15] R. Machleidt, Adv. Nucl. Phys. 19, 189 (1989).

[16] J. Strate et al., Nucl. Phys. A501, 51 (1989).

[17] G. Rauprich, S. Lemaitre, P. Niessen, K.R. Nyga, R. Reckenfelderbäumer, L. Sydow, H. Paetz gen. Schieck, H. Witala, and W. Glöckle, Nucl. Phys. A535, 313 (1991).

[18] R. Bieber et al., Phys. Rev. Lett. 84, 606 (2000).

[19] C. Hajduk, P.U. Sauer, and S.N. Yang, Nucl. Phys. A405, 605 (1983). 